\title{
Algoritmo MVMO-SH com Redimensionamento Populacional Adaptativo para Otimização de Filtros
}

\author{
Gabriel T. Ribeiro", Leandro dos S. Coelho, ${ }^{*, * *}$, Luiz Felipe Manke ${ }^{* * *}$, Viviana Cocco Mariani ${ }^{*, * * * *}$ \\ * Departamento de Engenharia Elétrica, Universidade Federal do Paraná, Curitiba, PR, Brasil \\ ${ }^{* * *}$ Programa de Pós-Grad. em Eng. de Produção (PPGEPS), Pontifícia Universidade Católica do Paraná, Curitiba, PR, Brasil \\ ***WEG Equipamentos Elétricos S.A., Departamento de Pesquisa e Inovação Tecnológica, Jaraguá do Sul, SC, Brasil \\ Programa de Pós-Graduação em Engenharia Mecânica, Pontifícia Universidade Católica do Paraná, Curitiba, PR, Brasil
}

(gabrielribeiro.ee@gmail.com,leandro.coelho@pucpr.br,luizmanke@gmail.com,viviana.mariani@pucpr.br)

\begin{abstract}
Several engineering problems are still not solved or there aren't enough hardware, software or theories capable of a viable solution. With technology advances and metaheuristics algorithms development, which search solutions inspired in the way nature deals with their problems, such problems may have a solution found. In this context there exists the Mean-Variance Mapping Optimization algorithm, which originally operates over a single solution but has a hybrid variant based in swarm intelligence which incorporates local search and multi-parent's crossover strategies and belong to the class of Differential Evolution, an evolutionary method which has shown to be competitive in practical optimization problems but at the same time is pretty sensitive to control parameters. To avoid the tuning of the algorithms control parameters there become algorithms with adaptive parameters. This paper proposes a variant of Mean-Variance Mapping Optimization with adaptive population size and its performance is assessed at a second order active filter optimization problem.
\end{abstract}

Resumo: Diversos problemas de engenharia não possuem soluções ou não existem infraestrutura de hardware, software ou teorias que viabilizem sua solução. Com o avanço da tecnologia e com o desenvolvimento de metaheurísticas, que buscam soluções inspiradas em comportamentos encontrados na natureza, surgem algoritmos que podem solucionar tais problemas. Um destes algoritmos é o MeanVariance Mapping Optimization, o qual originalmente opera sobre uma única solução mas possui uma variante híbrida, baseada em inteligência de partículas que incorpora busca local e estratégias de recombinação de múltiplos pais, e se enquadram na classe de algoritmos de Evolução Diferencial, um método evolucionário que mostrou-se competitivo na otimização de problemas práticos, mas que é sensível ao ajuste dos parâmetros de controle. Para evitar o ajuste dos parâmetros de controle surgiram os algoritmos com parâmetros adaptativos. Este artigo propõe uma variante do algoritmo Mean-Variance Mapping Optimization com tamanho populacional adaptativo e seu desempenho é analisado em um problema de otimização de um filtro ativo de segunda ordem.

Keywords: Optimization, Active Filter, Evolutionary Algorithms, Differential Evolution.

Palavras-chaves: Otimização, Filtro Ativo, Algoritmos Evolutivos, Evolução Diferencial.

\section{INTRODUÇÃO}

Avanços na eficiência de computadores e a evolução de softwares de cálculo numérico levaram a um aperfeiçoamento da teoria, métodos e algoritmos de otimização que permitiu encontrar soluções para problemas complexos de serem solucionados (Antoniou e Lu, 2017). Dentre as abordagens surgiram paradigmas da computação evolutiva (evolucionária) que são inspirados nos mecanismos da evolução biológica e comportamento dos organismos vivos.

Um fluxograma básico para implementação de um algoritmo evolutivo é apresentado na Fig. 1. O primeiro passo é a geração de uma população inicial de soluções (indivíduos) e logo após o algoritmo entra em um ciclo iterativo com dois passos básicos. No primeiro passo as soluções são avaliadas e selecionadas e no segundo passo as soluções utilizam mecanismo para se reproduzem e algumas sofrem uma operação que resulta em uma variação. As iterações (gerações) continuam até que se atinja um critério de parada do algoritmo evolutivo. Neste contexto, os passos de adaptação e de busca local são opcionais (Zhang et al., 2011), pois dependem da abordagem adotada.

Na última década surgiu o algoritmo de otimização MeanVariance Mapping Optimization (MVMO) classificado como uma técnica de otimização estocástica baseada em solução única proposta por Erlich, Venayagamoorthy e Worowat (2010). Tal método usa uma transformação para mutação baseada na variância média do arquivo de soluções. Contudo o MVMO opera sobre uma única solução não podendo ser classificado como um algoritmo evolutivo.

Na sequência, Rueda e Erlich (2013) propuseram uma variante híbrida do MVMO baseada em inteligência de enxames e algoritmos evolutivos que incorpora busca local e 
estratégias de recombinação de múltiplos pais, de forma a aumentar a diversidade da busca mantendo o compromisso entre exploração local e global, denominado MVMO-SH, um algoritmo evolutivo que opera sobre uma população de indivíduos.

O algoritmo MVMO possui algumas aplicações em engenharia, como na partição da rede elétrica de Québec em que se mostrou mais eficiente do que os outros algoritmos comparados (Sahoo e Sahoo, 2010). O MVMO também foi aplicado para identificação de um modelo de misturas Guassianas da carga no sistema elétrico Venezuelano em (Gonzalez-Longatt e Rueda, 2014), no problema de otimização do planejamento da expansão de um sistema de transmissão (Pringles e Rueda, 2012) e na otimização do despacho de carga ótimo (Shouman et al., 2017). Já Erlich, et al. (2014) investigaram o comportamento do MVMO-SH na resolução dos problemas da competição CEC 2014 e verificaram a capacidade de busca para diferentes problemas com diferentes dimensões e propriedades matemáticas.

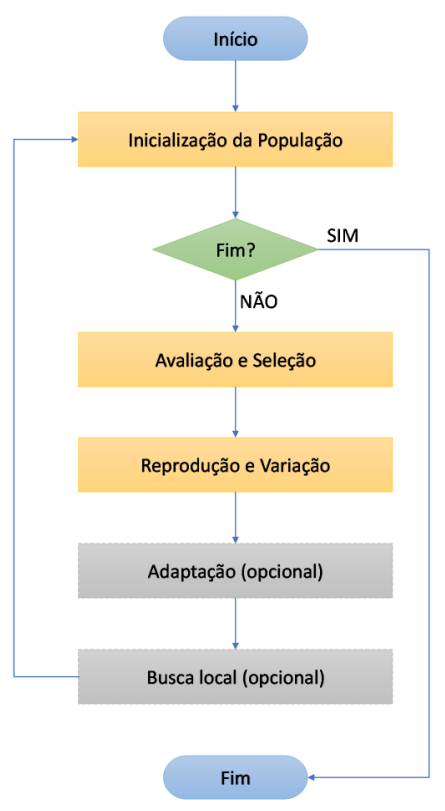

Fig. 1 Fluxograma básico de uma abordagem de computação evolutiva. Adaptado de (Zhang et al., 2011).

Uma abordagem eficiente de algoritmo evolutivo é a Evolução Diferencial (ED) que foi proposta inicialmente em Storn e Price (1997). A ED se mostrou competitiva na otimização de problemas práticos quando comparada aos métodos de otimização existentes, apesar de sua simplicidade. Contudo, seu desempenho é sensível ao ajuste dos parâmetros de controle. Para evitar o ajuste dos parâmetros de controle surgiram os algoritmos de evolução diferencial com parâmetros adaptativos, sendo um deles o LSHADE proposto por Tanabe e Fukunaga (2014) que usa um método de redução populacional monotônica reduzindo a população linearmente, denominado de estratégia de redução linear da dimensionalidade populacional Linear Population Reduction Strategy (LPRS) para modificar o algoritmo de otimização SHADE (Tanabe e Fukunaga, 2013).
Este artigo propõe a modificação do algoritmo MVMO-SH com a estratégia LPRS para adaptação do tamanho populacional de forma a incrementar sua habilidade de transição entre exploração global e local. Serão empregados o algoritmo MVMO original, o MVMO-SH e o algoritmo proposto a um problema de otimização de um filtro ativo e verificado seu desempenho através da curva de convergência e estatísticas de otimização.

As seguintes seções do artigo do artigo estão estruturadas da seguinte forma. Na seção 2 são apresentados os fundamentos da abordagem de otimização proposta. Após, na seção 3 e 4 são apresentados os materiais e métodos e análise de resultados, respectivamente. $\mathrm{Na}$ seção 5 é apresentada a conclusão e perspectiva de futura pesquisa.

\section{ABORDAGEM DE OTIMIZAÇÃO}

A seguir são apresentados os fundamentos do algoritmo MVMO-SH e da estratégia LPRS.

\section{$2.2 \mathrm{MVMO}-\mathrm{SH}$}

O algoritmo de otimização MVMO, Mean-Variance Mapping Optimization, é uma técnica de otimização estocástica baseada em solução única proposta por Erlich, Venayagamoorthy e Worowat (2010) que usa uma transformação para mutação inspirada na variância média do arquivo de soluções.

Rueda e Erlich (2013) propuseram uma variante híbrida do MVMO baseada em inteligência de partículas e que incorpora busca local e estratégias de recombinação de múltiplos pais, de forma a aumentar a diversidade da busca mantendo o compromisso entre exploração local e global, denominado MVMO-SH.

O fluxograma do MVMO-SH é exibido na Fig. 2. Inicialmente se definem os parâmetros do algoritmo e se inicializa a população uniformemente aleatória no intervalo $[0,1]$, que é o espaço de busca em que o algoritmo opera.

Para cada partícula sorteia-se um número aleatório, que caso seja inferior a uma constante $\gamma$, executa-se uma busca local senão avalia-se a função custo da partícula $k$. O número de avaliações, $i$, da função objetivo é atualizado para cada caso.

Logo após, a nova solução é posicionada no arquivo individual da partícula de acordo com a classificação de sua respectiva avaliação da função custo. Se tamanho do arquivo é superado excluem-se as piores soluções. Na sequência classificam-se as partículas em "boas ou ruins" e é realizada a seleção dos pais para a operação de recombinação.

Para cada partícula considerada "ruim" recombinam-se os pais selecionados a partir de um subconjunto das partículas "boas" de acordo com

$\boldsymbol{x}_{p}^{p a i}=\boldsymbol{x}_{k}+\beta\left(\boldsymbol{x}_{i}-\boldsymbol{x}_{j}\right)$

onde $\boldsymbol{x}$ representa uma solução, $p$ representa uma partícula ruim, $\beta$ é um número aleatório que é sorteado até que (1) resulte em um número no intervalo $[0,1], \boldsymbol{x}_{i}$ é a melhor 
solução global, $\boldsymbol{x}_{j}$ é a pior partícula dentre as boas e $\boldsymbol{x}_{k}$ é escolhida aleatoriamente entre as partículas boas. No caso de partículas boas elas recebem o valor do melhor pai local.

A geração da prole herda os valores de $\boldsymbol{x}_{p}^{p a i}$ e substitui $m$ dimensões pré-selecionadas de acordo com algum método (tal como roleta) pelos seus respectivos mapeamentos baseados na média e na variância do arquivo individual.

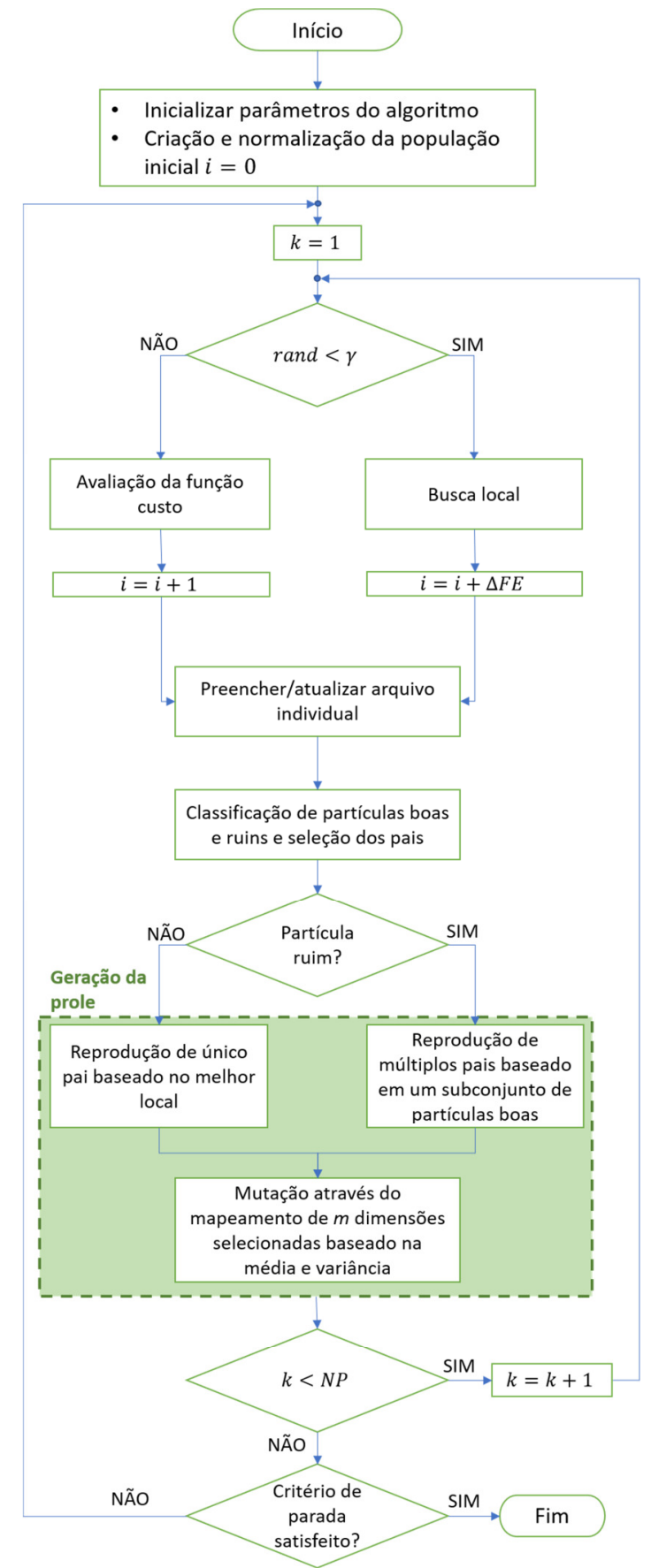

Fig. 2 Algoritmo MVMO-SH.

A proporção de partículas boas é determinada dinamicamente de acordo com

$$
\begin{aligned}
& G P=\left\lceil\left|N P \cdot g_{p}^{*}\right|-0.5\right\rceil \\
& g_{p}^{*}=g_{p_{\text {ini }}}^{*}+\alpha\left(g_{p_{\text {final }}^{*}}^{*}-g_{p_{\text {ini }}}^{*}\right)
\end{aligned}
$$

$\alpha=i / i_{\text {final }}$

onde arredondamento aproxima o número inteiro mais próximo, GP é o número de partículas "boas", $N P$ é o número de partículas, $g_{p}^{*}$ representa o limiar entre partículas boas e ruins, $g_{p_{\text {final }}}^{*}$ é o valor limiar que se terá ao final da otimização e $g_{p_{i n i}}^{*}$ é o valor limiar que se terá ao início da otimização, $\alpha$ é um fator de redução linear, $i$ é a iteração atual e $i_{\text {final }}$ é a iteração final.

\subsection{Estratégia LPRS}

A taxa de convergência dos algoritmos evolutivos é diretamente influenciada pelo tamanho da população. Pequenas populações implicam em uma rápida convergência ao mesmo tempo em que aumenta o risco de convergência para um ótimo local. Por outro lado, grandes populações permitem explorar uma grande área do espaço de busca, contudo tornam a convergência lenta (Tanabe e Fukunaga, 2014).

Tanabe e Fukunaga (2014) propuseram a utilização de um método de redução populacional monotônica que reduz a população linearmente, denominado de estratégia de redução linear da dimensionalidade populacional Linear Population Reduction Strategy (LPRS) para modificar o algoritmo de otimização SHADE (Tanabe e Fukunaga, 2013). A estratégia LPRS tem como vantagem a simplicidade e a necessidade de determinar apenas um parâmetro, que é o tamanho mínimo da população $N_{\min }$. A redução da população inicial $N_{\text {init }}$ se dá de acordo com

$N_{\text {novo }}=\left\lceil\left|\alpha\left(N_{\text {min }}-N_{\text {init }}\right)+N_{\text {init }}\right|-0.5\right\rceil$

\subsection{Otimização de Filtros Digitais}

Um filtro digital é um tipo de circuito que utiliza amplificadores operacionais em conjunto com elementos passivos (i.e., resistores, capacitores e indutores) para implementar uma função de resposta em frequência desejada e sua principal vantagem é que o amplificador previne que a impedância do próximo estágio influencie o estágio anterior.

A configuração de estágios com amplificadores em série permite aumentar a ordem do filtro e consequentemente a quantidade de polos e zeros que podem ser representadas na função de resposta em frequência.

De acordo com Magele e Ebner (2019) um filtro digital de segunda ordem pode ser descrito por

$A(P)=\frac{d_{0}+d_{1} P+d_{2} P^{2}}{c_{0}+c_{1} P+c_{2} P^{2}}$

onde $A$ é a amplitude de saída, $P$ é a variável complexa $j \Omega$ e $c$ e $d$ são os parâmetros do filtro. A função objetivo que deve ser minimizada é dada por,

$f(\boldsymbol{x})=\sum_{i=1}^{m}\left(A\left(j \Omega_{i}\right)-A\left(j \Omega_{i}\right)_{0}\right)^{2}$ 
onde $m$ é o total de amostras, $\boldsymbol{x}$ é o vetor $\left[d_{0}, d_{1}, d_{2}, c_{0}, c_{1}, c_{2}\right], A\left(j \Omega_{i}\right)$ é a saída do filtro e $A\left(j \Omega_{i}\right)_{0}$ é a saída de amplitude desejada.

\section{MATERIAIS E MÉTODOS}

Da mesma forma que o SHADE foi modificado com a inclusão da estratégia LPRS dando origem ao L-SHADE (Tanabe e Fukunaga, 2014), este artigo propõe a modificação do algoritmo MVMO-SH com a inclusão da estratégia LPRS, o qual denominamos de MVMO-LPRSH. O fluxograma do algoritmo proposto é mostrado na Fig 3.

$\mathrm{O}$ algoritmo é iniciado da mesma forma que no MVMO-SH com a diferença que, a cada nova iteração é calculado um novo valor para o tamanho da população com base na porcentagem do número máximo de avaliações da função objetivo ou de gerações de acordo com (5). Caso o valor $N_{\text {novo }}$ não seja menor do que o tamanho da população atual, o que é possível já que a redução precisa ser em passos de números inteiros, procede-se com o algoritmo MVMO-SH, caso contrário atualiza-se o tamanho da população excluindose os piores indivíduos.

Os algoritmos foram testados em um problema de otimização de um filtro digital de segunda ordem apresentado por Magele e Ebner (2019), tal que as entradas $\Omega$ e as amplitudes de saída desejadas possuam os valores apresentados na Tabela 1.

Tabela 1. Valores desejados de amplitude para cada $\Omega$.

\begin{tabular}{ll}
\hline$\Omega$ & Amplitude \\
\hline 0,01 & 0,9979535 \\
0,02 & 0,9918559 \\
0,03 & 0,9818305 \\
0,04 & 0,9680750 \\
0,05 & 0,9508510 \\
0,06 & 0,9304709 \\
0,07 & 0,9072836 \\
0,08 & 0,8816599 \\
0,09 & 0,8539798 \\
0,1 & 0,8246211 \\
0,2 & 0,5099020 \\
0,3 & 0,3374110 \\
0,4 & 0,4472136 \\
0,5 & 0,6695341 \\
0,6 & 0,9055385 \\
0,7 & 1,1383537 \\
0,8 & 1,3659730 \\
0,9 & 1,5889323 \\
1 & 1,8081014 \\
2 & 3,9010027 \\
3 & 5,9336300 \\
4 & 7,9501251 \\
5 & 9,9600640 \\
6 & 11,9667037 \\
7 & 13,9714519 \\
8 & 15,9750156 \\
9 & 17,9777888 \\
\hline
\end{tabular}

Foram comparados os algoritmos MVMO, MVMO-SH e MVMO-LPRSH, com 50 rodadas de otimização limitadas a 20000 avaliações da função objetivo. Os limites inferiores $\left(L_{B}\right)$ e superiores $\left(U_{B}\right)$ foram estabelecidos tal que

$$
\begin{aligned}
& L_{B}=[-10,-10,-10,0,0,0] \\
& U_{B}=[10,10,10,15,10,10]
\end{aligned}
$$

O tamanho do arquivo individual adotado foi 4, o tamanho mínimo da população $N_{\min }$ foi $5, g_{p_{\text {ini }}}^{*}$ foi 0,9 e $g_{p_{\text {final }}}^{*}$ foi 0,2 . Foram usadas 60 partículas para o tamanho da população do MVMO-SH assim como para a população inicial do MVMO-LPRSH. A seleção de variáveis para mutação foi aleatória, o número inicial de variáveis para mutação foi ajustado em 2 e o número final foi ajustado em 1, com decaimento linear, o fator de escala de forma foi ajustado em 1, o fator de escala da forma alternativa foi ajustado com início em 1 e final em 0,05 .

\section{RESULTADOS}

A curva de convergência para os três algoritmos é apresentada na Fig. 4. Percebe-se que inicialmente os três algoritmos convergem da mesma forma, a partir de um certo ponto, aproximadamente 4000 avaliações da função objetivo, o algoritmo MVMO parece estagnar em um mínimo local, o algoritmo MVMO-SH se aproveitando de sua maior capacidade de exploração de global pela inteligência de partículas consegue escapar do mínimo local. Apesar da melhora do MVMO-SH com relação ao MVMO, o algoritmo proposto MVMO-LPRSH converge para um valor ótimo global melhor do que os outros dois algoritmos.

A estatística da otimização é apresentada na Tabela 2. Todos os índices indicam uma melhora de desempenho partindo do pior desempenho com o MVMO, seguindo para MVMO-SH e o melhor desempenho sendo obtido pelo algoritmo proposto MVMO-LPRSH.

Tabela 2. Resultados da otimização

\begin{tabular}{llll}
\hline $\begin{array}{l}\text { Medidas } \\
\text { estatísticas }\end{array}$ & MVMO & MVMO-SH & $\begin{array}{l}\text { MVMO- } \\
\text { LPRSH }\end{array}$ \\
\hline $\begin{array}{l}\text { Mínimo } \\
\text { Maximo }\end{array}$ & $2,31 \cdot 10^{-3}$ & $591 \cdot 10^{-6}$ & $94,7 \cdot 10^{-6}$ \\
Média & $308 \cdot 10^{-3}$ & 0,45 & 0,08 \\
Mediana & $230 \cdot 10^{-3}$ & $5,15 \cdot 10^{-3}$ & $4,72 \cdot 10^{-3}$ \\
Desvio Padrão & $350 \cdot 10^{-3}$ & $64,8 \cdot 10^{-3}$ & $12,0 \cdot 10^{-3}$ \\
\hline
\end{tabular}




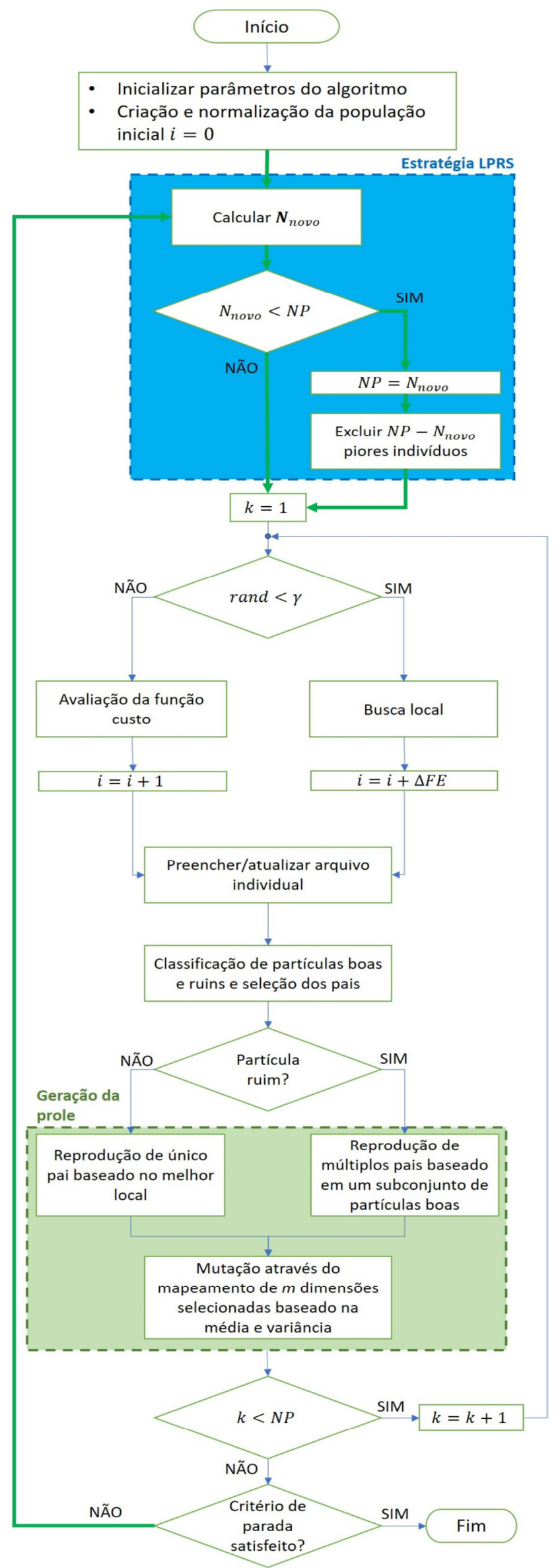

Fig. 3 Algoritmo MVMO-LPRSH.

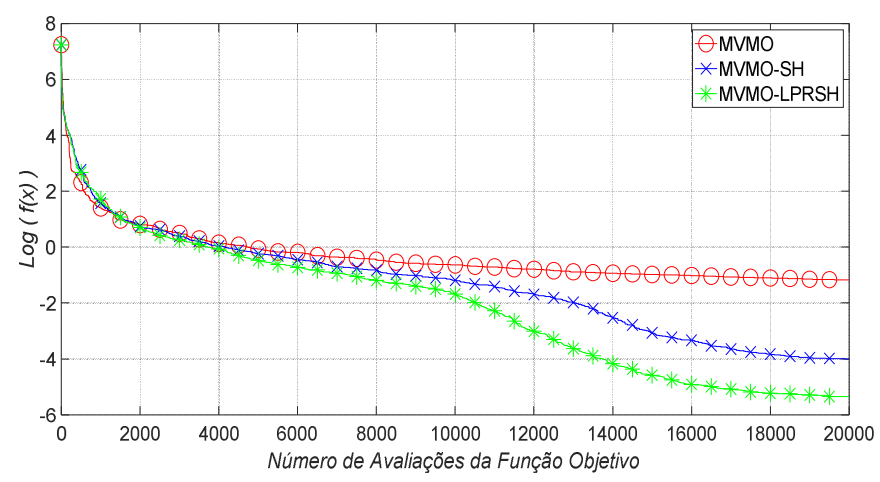

Fig. 4 Comportamento de convergência.

\section{CONCLUSÃO E FUTURA PESQUISA}

Foi apresentado o novo algoritmo de otimização proposto neste trabalho MVMO-LPRSH, resultante da implementação da estratégia de adaptação do tamanho populacional LPRS ao MVMO-SH, ao problema de otimização dos parâmetros de um filtro ativo de segunda ordem. A curva de convergência média após 50 repetições mostrou que o MVMO-LPRSH obtém um desempenho melhor do que os outros algoritmos da literatura usado para o mesmo problema teste.

O algoritmo MVMO por ser baseado em solução única é propenso a estagnar em mínimos locais, contudo o MVMOSH tem menor probabilidade desta estagnação pois explora a relação entre os membros da população na busca do ótimo global. Adicionalmente, o MVMO-LPRS demonstrou que a adaptação da dimensão populacional proporciona um desempenho ainda melhor. É preciso destacar que o uso de metaheurísticas de otimização não garante que será encontrado um ótimo global.

Consequentemente, a resposta em frequência do filtro ativo de segunda ordem projetado com os parâmetros definidos pelo MVMO-LPRS será muito mais próxima da característica esperada do que se fossem utilizados os parâmetros obtidos pelo MVMO ou MVMO-SH.

Em futuras pesquisas pretende-se estender a comparação para outros problemas de engenharia, problemas teste (tais como os de competições do IEEE CEC) e aperfeiçoar a adaptação dos demais hiperparâmetros de controle do algoritmo proposto neste artigo. Além disso, pretende-se estudar o impacto da variação da população inicial bem como o comportamento da redução da população ao longo das iterações.

\section{REFERÊNCIAS}

Antoniou, A., and Lu, W. (2007). Practical Optimization: Algorithms and Engineering Applications. Springer Science \& Business Media, USA.

Erlich, I., Venayagamoorthy, G.K., and Worawat, N. (2010). A mean-variance optimization algorithm. IEEE Congress on Evolutionary Computation, Barcelona, Spain.

Erlich, I., Rueda, J. L., Wildenhues, S., and Shewarega, F. (2014). Evaluating the Mean-Variance Mapping Optimization on the IEEE-CEC 2014 test suite. IEEE 
Congress on Evolutionary Computation (CEC), Beijing, China.

Gonzalez-Longatt, F. M., and Rueda, J. L. (2012). Identification of Gaussian mixture model using Mean Variance Mapping Optimization: Venezuelan case. IEEE PES Innovative Smart Grid Technologies Europe (ISGT Europe), Berlin, Germany.

Magele, C., and Ebner, T. (2019). Optimization in Electrical Engineering. [online] Available at: http://www.diegm.uniud.it/elettrotecnica/Didattica/Dispe nse/Dispense Corsi Brevi/Dispensa Magele Ottimizzazione.pdf [Accessed 14 Apr. 2019].

Pringles, R. M., and Rueda, J. L. (2012). Optimal transmission expansion planning using Mean-Variance Mapping Optimization. IEEE/PES Transmission and Distribution: Latin America Conference and Exposition ( $T \& D-L A)$, Montevideo, Uruguay.

Rueda, J.L., and Erlich, I. (2013). Hybrid mean-variance mapping optimization for solving the IEEE-CEC 2013 competition problems. IEEE Congress on Evolutionary Computation, Cancun, Mexico.

Sahoo, S, and Sahoo, S. R. (2010). Hybrid mean-variance mapping optimization to determine the number of clusters in network partitioning of Hydro Québec power grid. First International Conference on Networks \& Soft Computing, Guntur, India.

Shouman, N., Hegazi, Y. G., Krost, G., and Omran, W. A. (2017). Mean variance mapping optimization for solving economic load dispatch problem. IEEE Manchester PowerTech, Manchester, UK.

Storn, R., and Price, K. (1997). Differential evolution - a simple and efficient heuristic for global optimization over continuous spaces. Journal of Global Optimization, 11(4), 341-359.

Tanabe, R., and Fukunaga, A.S. (2013). Success-history based parameter adaptation for Differential Evolution. IEEE Congress on Evolutionary Computation, Cancun, Mexico.

Tanabe, R., and Fukunaga, A.S. (2014). Improving the search performance of SHADE using linear population size reduction. IEEE Congress on Evolutionary Computation, Beijing, China.

Zhang, J., Zhan, Z., Lin, Y., Chen, N., Gong, Y., Zhong, J., Chung, H., Li, Y., and Shi, Y. (2011). Evolutionary computation meets machine learning: A survey. IEEE Computational Intelligence Magazine, 6(4), 68-75. 